\title{
Os agravos psicossociais e a saúde mental da equipe de enfermagem na transcedência
} ao pós-pandemia de Covid-19

\author{
The psychosocial problems and the mental health of the nursing staff in transcending the post- \\ pandemic of Covid-19
}

Los problemas psicosociales y la salud mental del personal de enfermería en trascender la pospandémica del Covid-19

\author{
Carmen Lúcia de Araújo Paes \\ ORCID: https://orcid.org/0000-0001-9365-2451 \\ Fundação Santa Casa de Misericórdia do Pará, Brasil \\ E-mail: carmenaraujopaes@gmail.com \\ Ilma Pastana Ferreira \\ ORCID: https://orcid.org/0000-0002-9152-3872 \\ Fundação Santa Casa de Misericórdia do Pará, Brasil \\ E-mail: ilma.pastana@uepa.br \\ Amanda Ouriques de Gouveia \\ ORCID: https://orcid.org/0000-0002-6874-8352 \\ Fundação Santa Casa de Misericórdia do Pará, Brasil \\ E-mail: mandinhaouriques@hotmail.com \\ Valeria Regina Cavalcante dos Santos \\ ORCID: https://orcid.org/0000-0002-1264-8125 \\ Fundação Santa Casa de Misericórdia do Pará, Brasil \\ E-mail: valregsantos@ hotmail.com
}

\begin{abstract}
Resumo
Os aumentos dos índices de agravos psicossociais veem afetando a saúde mental da equipe de saúde, e esse acontecimento são atribuí́dos ao atual cenário vivido na pandemia do Covid-19 desde março de 2020, sendo os profissionais de enfermagem mais atingidos. Tem como objetivo analisar estudos sobre o adoecer dos trabalhadores da saúde, em especial os da equipe de enfermagem e a forma como eles interagem com o trabalho na transcendência ao pós-pandemia do Covid-19. Trata-se de uma revisão integrativa da literatura de natureza quantitativa e descritiva. A equipe de saúde em especial a de enfermagem está vulnerável para desenvolver frustações e insegurança no trabalho, principalmente neste cenário de pandemia do Covid-19, resultando aumento no índice de agravos psicossociais como depressão, ansiedade, estresse, distúrbio do sono, fadiga, desgaste físico e mental e outros, comprometendo a saúde mental desses profissionais. Desse modo, como agenda de pesquisa alternativa, sugerem-se as seguintes indagações: a) analisar os benefícios e deletérios da Covid-19 no psicológico da equipe de enfermagem; b) observar os agravos psicossociais e inadequações nos atendimentos de profissionais com agravos psicossociais pela pandemia; c) avaliar a eficácia do suporte oferecido a saúde desses profissionais, assim como, a articulação desta assistência.
\end{abstract}

Palavras-chave: Saúde mental; Terapia; Universidade.

\begin{abstract}
Increases in the rates of psychosocial problems have been affecting the mental health of the health team, and this event is attributed to the current scenario experienced in the Covid-19 pandemic since March 2020, with nursing professionals being the most affected. It aims to analyze studies on the illness of health workers, especially those of the nursing team and the way they interact with work in transcending the post-pandemic of the Covid-19. It is an integrative review of the literature of a quantitative and descriptive nature. The health team, in particular the nursing team, is vulnerable to developing frustration and job insecurity, especially in this cenario of the Covid-19 pandemic, resulting in an increase in the rate of psychosocial problems such as depression, anxiety, stress, sleep disturbance, fatigue, wear and tear physical and mental and others, compromising the mental health of these professionals. Thus, as an alternative research agenda, the following questions are suggested: a) to analyze the benefits and deleteries of Covid-19 in the psychological of the nursing team; b) observe the psychosocial problems and inadequacies in the attendance of professionals with psychosocial problems due to the pandemic; c) evaluate the effectiveness of the support offered to the health of these professionals, as well as the articulation of this assistance.
\end{abstract}

Keywords: Mental health; Health team; Pandemic; Covid-19; Psychosocial disorders. 


\begin{abstract}
Resumen
Los incrementos en las tasas de problemas psicosociales vienen afectando la salud mental del equipo de salud, y este evento se atribuye al escenario actual vivido en la pandemia Covid-19 desde marzo de 2020, siendo los profesionales de enfermería los más afectados. Tiene como objetivo analizar los estudios sobre la enfermedad de los trabajadores de la salud, especialmente los del equipo de enfermería y la forma en que interactúan con el trabajo en la trascendencia de la pos-pandémica de la Covid-19. Es una revisión integradora de la literatura de carácter cuantitativo y descriptivo. El equipo de salud, en particular el equipo de enfermería, es vulnerable a desarrollar frustración e inseguridad laboral, especialmente en este escenario de la pandemia del Covid-19, resultando en un aumento en la tasa de problemas psicosociales como depresión, ansiedad, estrés, alteración del sueño, fatiga, desgaste físico y mental y otros, comprometiendo la salud mental de estos profesionales. Así, como agenda alternativa de investigación, se plantean las siguientes cuestiones: a) analizar los beneficios y perjuicios del Covid-19 en la psicología del equipo de enfermería; b) observar los problemas psicosociales y las deficiencias en la atención de profesionales con problemas psicosociales debido a la pandemia; c) evaluar la efectividad del apoyo brindado a la salud de estos profesionales, así como la articulación de esta asistencia.
\end{abstract}

Palabras clave: Salud mental; Equipo de salud; Pandemia; Covid-19; Trastornos psicosociales.

\title{
1. Introdução
}

Segundo estudo de Catton et al. (2020), em dezembro de 2019, a cidade de Wuhan, capital da província de Hubei, na China, tornou-se o centro de um surto de pneumonia de causa desconhecida, ligada epidemiologicamente ao mercado local de frutos do mar. Em 07 de janeiro de 2020, os cientistas chineses isolaram um novo membro da família dos coronavírus, causador da Síndrome Respiratória Aguda Grave (SRAG), sendo em seguida designado de Doença do Coronavírus 2019 (Covid-19), em fevereiro de 2020, pela Organização Mundial da Saúde (OMS).

Nesse cenário no início de março de 2020, foram estabelecidas medidas de isolamento social e quarentena para diminuir o impacto da contaminação. As recomendações foram decretadas determinadas por se tratar de uma infecção viral aguda, altamente transmissível, cujo contágio se dá, principalmente, pelas vias respiratórias por meio da inalação de gotículas ou aerossóis no contato direto entre as pessoas e objetos contaminados pelo vírus (Moreira \& Lucca, 2020).

Aproximadamente $20 \%$ dos casos notificados são sintomáticos e podem cursar desde sinais e sintomas gripais leves, como febre e tosse, até quadros clínicos respiratórios graves com Lesão Pulmonar Aguda (LPA) e a síndrome do desconforto respiratório agudo (SDRA) com risco iminente de morte. Ademais, algumas pessoas podem manifestar sintomas gastrointestinais, como diarreia e vômito, infecções oculares, arritmias e lesão cardíaca aguda, insuficiência renal e disfunção hepática (Walton et al, 2020; El-Hage et al, 2020).

Portanto, tais acontecimentos impactaram as vidas dos indivíduos, chamando a atenção pelo alcance que teve e pela velocidade da sua disseminação. A partir disso, acometeu um coeficiente relativo da população, sobrecarregando o sistema de saúde global, sobretudo o Sistema Único de Saúde (SUS), no Brasil. Consequentemente, passou a exigir mais carga horária, desempenho, disponibilidade, qualificação, capacitação e princípios éticos dos profissionais de saúde (Ornel et al, 2020; Fofana et al, 2020).

Nesse contexto de combate ao novo coronavírus, Miranda et al (2020) afirma que os governos dão prioridade a saúde física, o combate e a prevenção da doença, com isso, as interferências a saúde mental tendem a ser minimizadas e subestimadas, o que pode gerar o aparecimento ou agravo de doenças mental já existentes (Miranda et al, 2020).

Já no que refere a saúde mental dos profissionais linha de frente da Covid-19, pesquisas atuais que compararam a pandemia do novo coronavírus com o surto de H1N1 e evidenciam que cerca de $80 \%$ dos trabalhadores que participaram do estudo possuem medo de atuar na pandemia da Covid-19. Levando isto em consideração e o fato da taxa mundial de infecção e mortalidade pela Sars-Cov-19 em trabalhadores da saúde está aumentando a cada dia, torna-se notável o risco ou agravo do adoecer mental nesse grupo (Hage et al, 2020).

Nesse cenário, as pesquisas em relação ao adoecimento dos profissionais da saúde e a relação que tem com o trabalho, assumem destaque nos estudos científicos, principalmente no aumento dos agravos ou riscos psicossociais desenvolvidos no 
período de pandemia e pós-pandemia da Covid-19. As ações de cuidar na área da saúde vão além dos procedimentos técnicos e conhecimento, envolvendo constante carga emocional dos profissionais da saúde para aliviar o sofrimento e lidar com as crises nas situações de desfechos negativos (Zannata \& Lucca, 2015).

Além disso, tais profissionais, geralmente, encontram-se submetidos a fatores relacionados à organização e precarização do trabalho, como a divisão e o parcelamento das tarefas, falta de reconhecimento profissional multifuncionalidade, exigência de produtividade, baixos salários e deficiências nas redes do sistema de saúde, o que podem desencadear agravos à saúde psicossocial (Carreiro et al, 2013).

Isto posto, considera-se que os agravos ou riscos psicossociais do trabalho está diretamente atrelado à forma como o trabalho é concebido, organizado ou gerido. Logo no pós-pandemia, os agentes nocivos associados especialmente à saúde mental do trabalhador poderão desencadear uma série de doenças mentais, principalmente, entre o grupo supracitado (Ornell et al, 2020).

Diante do exposto, o estudo teve como objetivo: analisar pesquisas sobre o adoecer dos trabalhadores de saúde, em especial a equipe de enfermagem e a forma como ele interage com o trabalho na transcendência ao pós-pandemia do Covid-19. Segundo as produções científicas sobre a transcendência ao pós-pandemia, propiciando uma compreensão mais ampla da ocorrência e do curso dos agravos à saúde, bem como das suas consequências diretas e indiretas para os indivíduos, famílias e sociedade.

\section{Metodologia}

Trata-se de uma revisão integrativa de literatura de natureza quantitativa e descritiva, realizada a partir da obtenção de dados efetuada por meio de fontes secundárias, este trabalho formulou-se a partir da seguinte questão norteadora: quais são os principais agravos psicossociais que atingem os profissionais da equipe de enfermagem na pós-pandemia do Covid-19?

Logo, ele aconteceu em 6 etapas, como demostra o Esquema 1, sendo que na primeira fase ocorreu a busca pelos artigos, onde indexou-se nas bases de dados online Scielo, Biblioteca Virtual de Saúde (BVS) e PubMed os seguintes descritores "saúde mental", "equipe de saúde", "pandemia", "Covid-19" e "agravos psicossociais" obtendo a partir disso 49 artigos.

Fora, então, empregado como critério de inclusão desses artigos pré-selecionados estudos com o recorte temporal de 2016 à 2020, redigidos em português, espanhol, inglês e francês, com delineamento qualitativo e descritivo de levantamento normativo ou metanálise de temáticas voltadas para a saúde mental e os agravos psicossociais na equipe de saúde hospitalar, em especial a equipe de enfermagem, pós-pandemia.

Como critérios de exclusão não se utilizou artigos que transcorriam sobre a saúde mental do profissional de saúde na atenção básica, os que não associavam os temas de saúde mental a pandemia do Covid-19, os que articulavam somente sobre o novo coronavírus, o qual discutia somente sobre a saúde mental da classe médica e os que não cabiam nos critérios de inclusão.

Em seguida, na segunda fase, desenvolveu-se a leitura dos resumos dos 49 artigos pré-selecionados, os quais 25 obedeciam aos critérios de inclusão e exclusão, esses foram selecionados e logo após lidos, permanecendo 16 artigos que se adequavam ao tema proposto. Posteriormente, realizou-se a terceira etapa com a análise dos estudos que ocorreu, por meio, da releitura exaustiva dos materiais selecionados e fichamento das partes mais pertinentes, assim conseguindo compreender as noções de todos os estudos em sua completude. Subsequentemente, aconteceu a quarta fase com a metanálise dos estudos que se deu através da comparação dos dados obtidos no fichamento durante a construção do artigo. 
Esquema 1. Organograma do processo de seleção dos artigos para este estudo.



Fonte: Pesquisa direta $(2020$

\section{Resultados}

O levantamento bibliográfico foi executado utilizando mecanismos de buscas online, tal método obteve após minuciosa investigação 16 artigos como já dito anteriormente, sendo que estes estudos foram agrupados de acordo com a sua temática: O suporte a saúde mental dos profissionais de saúde durante a pandemia, a saúde mental da equipe multiprofissional na pandemia, o papel da equipe de enfermagem na linha de frente e a saúde mental da equipe de enfermagem pós-pandemia de Covid-19.

Ressalta-se que todos os artigos foram retirados do ano de 2020, desses $43,75 \%$ são do mês de junho e $25,00 \%$ são do mês de abril. Já com relação ao idioma, 75,00\% estavam na língua inglesa, 18,75\% estavam em português e 6,25\% estavam em 
francês. Desses, cerca de 30\% dos artigos são chineses, como representado a Tabela 1.

Tabela 1. Caracterização dos artigos selecionados.

\begin{tabular}{|c|c|c|}
\hline Caracterização & Número de Estudos & $\%$ \\
\hline \multicolumn{3}{|l|}{ Mês de publicação } \\
\hline Janeiro & 1 & $6,25 \%$ \\
\hline Fevereiro & 0 & $0 \%$ \\
\hline Março & 1 & $6,25 \%$ \\
\hline Abril & 4 & $25,00 \%$ \\
\hline Maio & 1 & $6,25 \%$ \\
\hline Junho & 7 & $43,75 \%$ \\
\hline Julho & 1 & $6,25 \%$ \\
\hline Agosto & 0 & $0 \%$ \\
\hline Setembro & 1 & $6,25 \%$ \\
\hline \multicolumn{3}{|c|}{ Idioma de publicação } \\
\hline Inglês & 12 & $75,00 \%$ \\
\hline Português & 3 & $18,75 \%$ \\
\hline Francês & 1 & $6,25 \%$ \\
\hline \multicolumn{3}{|l|}{ País de publicação } \\
\hline Brasil & 3 & $18,75 \%$ \\
\hline EUA & 1 & $6,25 \%$ \\
\hline Espanha & 1 & $6,25 \%$ \\
\hline Reino Unido & 3 & $18,75 \%$ \\
\hline França & 1 & $6,25 \%$ \\
\hline China & 5 & $31,25 \%$ \\
\hline Itália & 1 & $6,25 \%$ \\
\hline Paquistão & 1 & $6,25 \%$ \\
\hline
\end{tabular}

Fonte: Pesquisa direta (2020).

No que diz respeito às plataformas de busca online, a mais utilizada foi a Pubmed com cerca de 9 nove artigos indexados, sendo seguida pela Biblioteca de Virtual de Saúde (BVS) com 5cinco artigos encontrados, e, por fim, a Scientific Electronic Library Online (Scielo) com 1 um artigo identificado, como demostra a Tabela 2.

Tabela 2. Organização dos estudos incluídos no acervo, de acordo com a plataforma de pesquisa.

\begin{tabular}{ccc} 
Nome do Periódico & Número de Estudos & Porcentagem \\
\hline PubMed & 9 & $56,25 \%$ \\
Biblioteca de Virtual de Saúde & 5 & $31,25 \%$ \\
Scientific Electronic Library Online & 1 & $6,25 \%$ \\
\hline Total & $\mathbf{1 6}$ & $\mathbf{1 0 0 \%}$ \\
\hline
\end{tabular}


Já no que refere aos principais agravos psicossociais citados na literatura em que as equipes de saúde que estavam na linha de frente contra o Covid-19, em especial a equipe de enfermagem, passou a enfrentar pós-pandemia, o qual obteve o maior número de citações foi a ansiedade precedida pela depressão, estresse e distúrbios no sono. Abaixo a Tabela 3 com a quantidade de citações encontradas de cada sintoma por artigo.

Tabela 3. Porcentagem de sintomas citados por artigos.

\begin{tabular}{ccc} 
Agravo psicossocial & $\begin{array}{c}\text { Número de artigos que o } \\
\text { sintoma foi citado }\end{array}$ & Porcentagem de citações \\
\hline Ansiedade & 8 & $50,00 \%$ \\
Depressão & 7 & $43,76 \%$ \\
Estresse & 6 & $37,50 \%$ \\
Distúrbios no sono & 5 & $31,25 \%$ \\
Fadiga & 4 & $25,00 \%$ \\
Desgaste mental e físico & 4 & $25,00 \%$ \\
Transtorno de estresse pós-traumático & 4 & $25,00 \%$ \\
Síndrome de Burnout & 3 & $18,75 \%$ \\
Vícios & 2 & $12,25 \%$ \\
\hline
\end{tabular}

Fonte: Pesquisa direta (2020).

De modo a demostrar com maior clareza a sinopse das 16 amostras selecionadas, foi construído o Quadro 1, o qual contém a identificação dos artigos pelos autores, objetivos do estudo, tipo de amostragem, metodologia de pesquisa, análise dos resultados e considerações científicas.

Quadro 1. Sinopse dos estudos selecionados.

\begin{tabular}{|c|c|c|c|c|c|}
\hline Autores & Objetivos & Amostra & Estudo/Método & Resultados & Considerações \\
\hline $\begin{array}{l}\text { Amanda } \\
\text { Moreira e } \\
\text { Sérgio Lucca }\end{array}$ & $\begin{array}{l}\text { Descrever e discutir } \\
\text { a atuação dos } \\
\text { profissionais de } \\
\text { enfermagem, sua } \\
\text { exposição aos } \\
\text { fatores de risco no } \\
\text { trabalho e a } \\
\text { importância do apoio } \\
\text { psicossocial na } \\
\text { pandemia da Covid- } \\
19 .\end{array}$ & 81 estudos. & $\begin{array}{l}\text { Trata-se de uma } \\
\text { revisão narrativa de } \\
\text { análise dos } \\
\text { conteúdos técnico- } \\
\text { científicos } \\
\text { produzidos em } \\
\text { diferentes países } \\
\text { acerca dos } \\
\text { profissionais de } \\
\text { enfermagem no } \\
\text { âmbito da } \\
\text { pandemia. }\end{array}$ & $\begin{array}{l}\text { Os afastamentos dos } \\
\text { colegas contaminados, as } \\
\text { altas demandas, a escassez } \\
\text { de materiais, o medo de ser } \\
\text { contaminado e a falta de } \\
\text { apoio psicossocial } \\
\text { sobrecarregam esses } \\
\text { profissionais e causa } \\
\text { estresse e pode desencadear } \\
\text { esgotamento físico e } \\
\text { psíquico. }\end{array}$ & $\begin{array}{l}\text { Considerando a } \\
\text { importância do trabalho da } \\
\text { equipe de enfermagem, } \\
\text { além das condições de } \\
\text { trabalho adequadas, o apoio } \\
\text { psicossocial na preservação } \\
\text { da saúde mental destes } \\
\text { profissionais é essencial } \\
\text { para os trabalhadores e para } \\
\text { a qualidade do cuidado } \\
\text { prestado. }\end{array}$ \\
\hline $\begin{array}{l}\text { Fernanda } \\
\text { Miranda et al. }\end{array}$ & $\begin{array}{l}\text { Refletir sobre as } \\
\text { condições de } \\
\text { trabalho dos } \\
\text { profissionais de } \\
\text { enfermagem no } \\
\text { enfrentamento ao } \\
\text { novo coronavírus e } \\
\text { apontar o impacto na } \\
\text { vida desses } \\
\text { profissionais em } \\
\text { meio à pandemia. }\end{array}$ & 24 estudos. & $\begin{array}{l}\text { Revisão sistêmica } \\
\text { da bibliográfica } \\
\text { com metanálise. }\end{array}$ & $\begin{array}{l}\text { As fragilidades encontradas } \\
\text { no cotidiano laboral dos } \\
\text { profissionais de } \\
\text { enfermagem são descritores } \\
\text { pela literatura nacional e } \\
\text { internacional, nas quais } \\
\text { estão excluídas as más } \\
\text { condições de trabalho, } \\
\text { sobrecarga física e mental, } \\
\text { baixa renumeração e } \\
\text { ausência de EPI's } \\
\text { adequados para o } \\
\text { enfrentamento desse }\end{array}$ & $\begin{array}{l}\text { A reflexão pode contribuir } \\
\text { para repensar a saúde e } \\
\text { segurança dos profissionais } \\
\text { de enfermagem visando } \\
\text { uma assistência com } \\
\text { qualidade e segurança aos } \\
\text { pacientes frente a esta } \\
\text { doença. }\end{array}$ \\
\hline
\end{tabular}




\begin{tabular}{|c|c|c|c|c|c|}
\hline & & & & agravo. & \\
\hline $\begin{array}{l}\text { Kristen } \\
\text { Santarone et } \\
\text { al. }\end{array}$ & $\begin{array}{l}\text { Discutir sobre as } \\
\text { formas de mantar a } \\
\text { resiliência mental } \\
\text { dos funcionários da } \\
\text { linha de frente, } \\
\text { oferecendo soluções } \\
\text { que lhes permitam } \\
\text { desempenhar suas } \\
\text { funções. }\end{array}$ & 10 estudos. & $\begin{array}{l}\text { Revisão sistêmica } \\
\text { da bibliográfica } \\
\text { com metanálise. }\end{array}$ & $\begin{array}{l}\text { Para adequar a necessidade } \\
\text { da comunidade com as } \\
\text { forças reserva de } \\
\text { profissionais de saúde } \\
\text { qualificados é preciso que } \\
\text { estes estejam ativos em } \\
\text { momentos de emergência } \\
\text { ou alto volume. }\end{array}$ & $\begin{array}{l}\text { Torna-se necessário tomar } \\
\text { medidas para preservar o } \\
\text { bem-estar de nossos } \\
\text { provedores de linha de } \\
\text { frente e protege-los de } \\
\text { infecções. }\end{array}$ \\
\hline $\begin{array}{l}\text { Felipe Ornell } \\
\text { et al. }\end{array}$ & $\begin{array}{l}\text { Identificar grupos } \\
\text { com alto risco de } \\
\text { desenvolver } \\
\text { problemas } \\
\text { emocionais, a fim de } \\
\text { monitorar sua saúde } \\
\text { mental e realizar } \\
\text { intervenções } \\
\text { psicológicas e } \\
\text { psiquiátricas } \\
\text { precoces. }\end{array}$ & 16 estudos. & $\begin{array}{l}\text { Revisão sistêmica } \\
\text { da bibliográfica } \\
\text { integrativa. }\end{array}$ & $\begin{array}{l}\text { Contata-se que a curva de } \\
\text { contágio da Covid-19 está } \\
\text { aumentando e as estratégias } \\
\text { de saúde precisam ser } \\
\text { intensificadas, requerendo } \\
\text { atenção primária geral aos } \\
\text { profissionais de saúde que } \\
\text { podem requerer diferentes } \\
\text { estratégias de informação, } \\
\text { apoio ou intervenção. }\end{array}$ & $\begin{array}{l}\text { O governo e as agências de } \\
\text { saúde têm a } \\
\text { responsabilidade de } \\
\text { proteger o bem-estar } \\
\text { psicológico da comunidade } \\
\text { de saúde em todo o mundo. }\end{array}$ \\
\hline $\begin{array}{l}\text { Muhammad } \\
\text { Sarwar e Huda } \\
\text { Sarwar }\end{array}$ & $\begin{array}{l}\text { Abordar a } \\
\text { capacidade de } \\
\text { automedicação entre } \\
\text { médicos durante o } \\
\text { sofrimento mental. }\end{array}$ & 04 estudos. & $\begin{array}{l}\text { Revisão sistêmica } \\
\text { da bibliográfica } \\
\text { integrativa. }\end{array}$ & $\begin{array}{l}\text { É perceptível que os } \\
\text { profissionais de saúde, já } \\
\text { sujeitos a burnout e estresse } \\
\text { mental, estão agora } \\
\text { expostos a um novo vírus } \\
\text { que os coloca em risco de } \\
\text { contrair uma doença fatal e } \\
\text { cria um estado de incerteza, } \\
\text { pânico e apreensão do seu } \\
\text { bem-estar e ao de sua } \\
\text { família. }\end{array}$ & $\begin{array}{l}\text { Portanto com a } \\
\text { implementação de } \\
\text { estratégias preventivas } \\
\text { contra a doença, as } \\
\text { disposições de apoio à } \\
\text { saúde mental de curto e } \\
\text { longo prazo, incluindo } \\
\text { primeiros socorros } \\
\text { psicológicos. }\end{array}$ \\
\hline E Liu Yu et al. & $\begin{array}{l}\text { Explorar as } \\
\text { experiências de } \\
\text { enfermeiras da linha } \\
\text { de frente no combate } \\
\text { à epidemia da } \\
\text { doença coronavírus } \\
\text { em } 2019 \text {. }\end{array}$ & $\begin{array}{l}15 \\
\text { enfermeiras } \\
\text { de primeira } \\
\text { linha que } \\
\text { cuidam de } \\
\text { pacientes } \\
\text { Covid-19 } \\
\text { foram } \\
\text { recrutadas de } \\
\text { dois hospitais } \\
\text { em Wuhan, } \\
\text { China, de } 26 \\
\text { de janeiro a } 5 \\
\text { de fevereiro } \\
\text { de } 2020 \text {. }\end{array}$ & $\begin{array}{l}\text { Os dados foram } \\
\text { coletados por meio } \\
\text { de entrevistas } \\
\text { individuais } \\
\text { semiestruturadas e } \\
\text { analisados usando } \\
\text { métodos } \\
\text { qualitativos padrão. }\end{array}$ & $\begin{array}{l}\text { Foi evidenciado o } \\
\text { enfrentando novos desafios } \\
\text { e perigos tremendos, a forte } \\
\text { pressão por medo de } \\
\text { infecção, cansaço por } \\
\text { cargas de trabalho pesadas e } \\
\text { estresse de enfermagem } \\
\text { pacientes com Covid-19 } \\
\text { gravemente enfermos, bem } \\
\text { como, o senso de dever e } \\
\text { identidade como provedor } \\
\text { de saúde e a compreensão } \\
\text { racional da epidemia. }\end{array}$ & $\begin{array}{l}\text { Embora o intenso trabalho } \\
\text { de resgate tenha esgotado } \\
\text { as enfermeiras da linha de } \\
\text { frente, tanto física quanto } \\
\text { emocionalmente, elas } \\
\text { mostraram um espírito de } \\
\text { dedicação e sentiram a } \\
\text { responsabilidade de superar } \\
\text { esta epidemia. }\end{array}$ \\
\hline $\begin{array}{l}\text { Ziwei Teng et } \\
\text { al. }\end{array}$ & $\begin{array}{l}\text { Investigar o impacto } \\
\text { da Covid-19 na } \\
\text { saúde mental do } \\
\text { pessoal da linha de } \\
\text { frente. }\end{array}$ & $\begin{array}{l}24 \\
\text { enfermeiros. }\end{array}$ & $\begin{array}{l}\text { Foram usados os } \\
\text { estudos padrões de } \\
\text { Paciente Health } \\
\text { Questionnare-9 } \\
\text { (PHQ-9), Escala de } \\
\text { Autoavaliação de } \\
\text { Ansiedade (SAS) e } \\
\text { Escala de } \\
\text { Autoavaliação de } \\
\text { Fadiga (FSAS). }\end{array}$ & $\begin{array}{l}\text { As taxas de detecção de } \\
\text { depressão, ansiedade e } \\
\text { fadiga foram } 49,1 \%, 21,8 \% \\
\text { e } 76,0 \% \text { entre os } \\
\text { funcionários da linha de } \\
\text { frente. As taxas de } \\
\text { depressão, ansiedade e } \\
\text { fadiga em trabalhadores } \\
\text { comunitários foram maiores } \\
\text { do que em trabalhadores } \\
\text { médicos e outras equipes } \\
\text { ocupacionais }(\mathrm{P}<0,01) \text {. }\end{array}$ & $\begin{array}{l}\text { Os trabalhadores da linha } \\
\text { de frente devem } \\
\text { administrar o trabalho e o } \\
\text { tempo de descanso de } \\
\text { maneira razoável para } \\
\text { ajustar seu humor negativo } \\
\text { e fadiga. }\end{array}$ \\
\hline $\begin{array}{l}\text { Emanuele } \\
\text { Preti et al. }\end{array}$ & $\begin{array}{l}\text { Fornecer evidências } \\
\text { quantitativas sobre o } \\
\text { impacto psicológico } \\
\text { de surtos epidêmicos } \\
\text { / pandêmicos. }\end{array}$ & 44 estudos. & $\begin{array}{l}\text { Revisão sistêmica } \\
\text { da bibliográfica } \\
\text { com metanálise. }\end{array}$ & $\begin{array}{l}\text { Apresentaram estresse pós- } \\
\text { traumático } 40 \% \text { dos } \\
\text { profissionais, seguidos de } \\
27,5 \% \text { com insônia e } 36,1 \% \\
\text { com sintomas de ansiedade. }\end{array}$ & $\begin{array}{l}\text { As evidências empíricas } \\
\text { sublinham a necessidade de } \\
\text { abordar os efeitos } \\
\text { prejudiciais de surtos de } \\
\text { epidemia / pandemia em } \\
\text { profissionais de saúde }\end{array}$ \\
\hline
\end{tabular}




\begin{tabular}{|c|c|c|c|c|c|}
\hline & & & & & mental. \\
\hline $\begin{array}{l}\text { Yuanyuan Mo } \\
\text { et al. }\end{array}$ & $\begin{array}{l}\text { Investigar o estresse } \\
\text { no trabalho entre } \\
\text { enfermeiras chinesas } \\
\text { que estão apoiando } \\
\text { Wuhan na luta } \\
\text { contra a infecção por } \\
\text { Doença por } \\
\text { Coronavírus } 2019 \\
\text { (Covid - 19) e } \\
\text { explorar os fatores } \\
\text { de influência } \\
\text { relevantes. }\end{array}$ & $\begin{array}{l}180 \\
\text { enfermeiros. }\end{array}$ & $\begin{array}{l}\text { Foram utilizadas } \\
\text { ferramentas de } \\
\text { coleta de dados de } \\
\text { Escala de } \\
\text { Sobrecarga de } \\
\text { Estresse (SOS) e a } \\
\text { Escala de } \\
\text { Ansiedade de } \\
\text { Autoavaliação } \\
\text { (SAS), além de } \\
\text { análises de } \\
\text { regressão múltipla } \\
\text { para explorar os } \\
\text { fatores de } \\
\text { influência. }\end{array}$ & $\begin{array}{l}\text { Os escores SOS }(39,91 \pm \\
12,92) \text { e SAS }(32,19 \pm 7,56) \\
\text { deste grupo de enfermeiros } \\
\text { foram positivamente } \\
\text { correlacionados }(\mathrm{p}<0,05), \\
\text { com isso, a análise de } \\
\text { regressão múltipla mostrou } \\
\text { que apenas crianças, horas } \\
\text { de trabalho por semana e } \\
\text { ansiedade foram os } \\
\text { principais fatores que } \\
\text { afetam o estresse da } \\
\text { enfermeira. }\end{array}$ & $\begin{array}{l}\text { Os chefes de enfermagem } \\
\text { devem estar atentos ao } \\
\text { estresse no trabalho e aos } \\
\text { fatores que influenciam os } \\
\text { enfermeiros que lutam } \\
\text { contra a infecção pelo } \\
\text { Covid - } 19 \text { e oferecer } \\
\text { soluções para manter a } \\
\text { saúde mental desses } \\
\text { enfermeiros. }\end{array}$ \\
\hline $\begin{array}{l}\text { Jianbo Lai et } \\
\text { al. }\end{array}$ & $\begin{array}{l}\text { Avaliar a magnitude } \\
\text { dos resultados de } \\
\text { saúde mental e } \\
\text { fatores associados } \\
\text { entre profissionais } \\
\text { de saúde que tratam } \\
\text { de pacientes } \\
\text { expostos ao Covid- } \\
19 \text { na China. }\end{array}$ & $\begin{array}{l}1.257 \\
\text { profissionais } \\
\text { da saúde. }\end{array}$ & $\begin{array}{l}\text { Estudo transversal, } \\
\text { baseado em } \\
\text { pesquisa e } \\
\text { estratificado por } \\
\text { região coletou } \\
\text { dados } \\
\text { demográficos e } \\
\text { medições de saúde } \\
\text { mental, em } 34 \\
\text { hospitais de } 29 \text { de } \\
\text { janeiro de } 2020 \text { a } 3 \\
\text { de fevereiro de } \\
\text { 2020, na China. }\end{array}$ & $\begin{array}{l}\text { O grau de sintomas de } \\
\text { depressão, ansiedade, } \\
\text { insônia e angústia foi } \\
\text { avaliado pelas versões } \\
\text { chinesas do Questionário de } \\
\text { Saúde do Paciente de } 9 \\
\text { itens, a escala de Transtorno } \\
\text { de Ansiedade Generalizada } \\
\text { de } 7 \text { itens, o Índice de } \\
\text { Gravidade de Insônia de } 7 \\
\text { itens, e a escala revisada de } \\
22 \text { itens da escala de } \\
\text { impacto do evento, } \\
\text { respectivamente. }\end{array}$ & $\begin{array}{l}\text { Nesta pesquisa os } \\
\text { participantes relataram } \\
\text { experiência de carga } \\
\text { psicológica, especialmente } \\
\text { enfermeiras, mulheres, } \\
\text { aquelas em Wuhan e na } \\
\text { linha de frente profissionais } \\
\text { de saúde diretamente } \\
\text { envolvidos no diagnóstico, } \\
\text { tratamento e cuidado de } \\
\text { pacientes com COVID-19. }\end{array}$ \\
\hline Tawfiq e & $\begin{array}{l}\text { Destacar a } \\
\text { importante questão } \\
\text { do bem-estar } \\
\text { psicológico dos } \\
\text { profissionais de } \\
\text { saúde durante o surto } \\
\text { de Covid-19. }\end{array}$ & $\begin{array}{l}106 \\
\text { profissionais } \\
\text { da saúde. }\end{array}$ & $\begin{array}{l}\text { Pesquisa voluntária } \\
\text { e anônima com a } \\
\text { equipe em um } \\
\text { centro cardíaco } \\
\text { terciário, por meio, } \\
\text { da aplicação de } \\
\text { questionário com } \\
\text { a escala de } \\
\text { avaliação de } \\
\text { depressão do } \\
\text { Patient Health } \\
\text { Questionnaire } \\
\text { (PHQ-9) e a Escala } \\
\text { de Estresse } \\
\text { Percebido-4 (PSS- } \\
\text { 4) para avaliar os } \\
\text { níveis de estresse. }\end{array}$ & $\begin{array}{l}\text { Foi contatado uma } \\
\text { revalência, no momento } \\
\text { atual, de depressão leve, } \\
\text { aumento dos níveis de } \\
\text { estresse e ansiedade leve } \\
\text { entre os profissionais de } \\
\text { saúde que trabalharam } \\
\text { durante a atual pandemia de } \\
\text { Covid-19. }\end{array}$ & $\begin{array}{l}\text { O reconhecimento precoce } \\
\text { de sinais de sofrimento } \\
\text { psicológico, a criação de } \\
\text { serviços de suporte } \\
\text { adequados e os cuidados } \\
\text { posteriores de longo prazo } \\
\text { para nossos profissionais } \\
\text { de saúde são de suma } \\
\text { importância. }\end{array}$ \\
\hline $\begin{array}{l}\text { Nina Fofana et } \\
\text { al. }\end{array}$ & $\begin{array}{l}\text { Sintetizar o dilema } \\
\text { da doença mental } \\
\text { como resultado de } \\
\text { uma pandemia e } \\
\text { inicia sugestões para } \\
\text { ajudar o público em } \\
\text { geral, os } \\
\text { profissionais de } \\
\text { saúde. }\end{array}$ & 19 estudos. & $\begin{array}{l}\text { Revisão sistêmica } \\
\text { da bibliográfica } \\
\text { com metanálise. }\end{array}$ & $\begin{array}{l}\text { A literatura sobre saúde } \\
\text { mental e Covid-19 é escassa } \\
\text { no momento, a existente } \\
\text { indica que depressão e } \\
\text { PTSD, PTSS e TOC, } \\
\text { aumentaram } \\
\text { significativamente devido } \\
\text { ao Covid-19. }\end{array}$ & $\begin{array}{l}\text { Sugerir pesquisas } \\
\text { adicionais para analisar a } \\
\text { saúde mental e as } \\
\text { consequências } \\
\text { neuropsiquiátricas, } \\
\text { abordando os cuidados de } \\
\text { saúde mental, o tratamento } \\
\text { e a adoção de medidas } \\
\text { preventivas durante a } \\
\text { pandemia Covid-19. }\end{array}$ \\
\hline $\begin{array}{l}\text { Holly Blake et } \\
\text { al. }\end{array}$ & $\begin{array}{l}\text { Abordar os impactos } \\
\text { da Covid-19 na } \\
\text { saúde mental, } \\
\text { protegendo e } \\
\text { promovendo o bem- } \\
\text { estar psicológico dos } \\
\text { profissionais de } \\
\text { saúde durante e após } \\
\text { o surto. }\end{array}$ & 97 estudos. & $\begin{array}{l}\text { Método Agile em } 3 \\
\text { etapas, com } \\
\text { atividades de } \\
\text { envolvimento } \\
\text { público, conteúdo e } \\
\text { desenvolvimento } \\
\text { por pares e entrega } \\
\text { e avaliação. }\end{array}$ & $\begin{array}{l}\text { O pacote de suporte digital } \\
\text { sobre 'bem-estar } \\
\text { psicológico para } \\
\text { profissionais de saúde', } \\
\text { contabilizou } 97 \text { pontos na } \\
\text { primeira etapa, seguido de } \\
10 \text { e } 55 \text { pontos, na segunda } \\
\text { e terceira etapa, } \\
\text { respectivamente. }\end{array}$ & $\begin{array}{l}\text { Recomenda-se o } \\
\text { fornecimento deste pacote } \\
\text { eletrônico aos profissionais } \\
\text { de saúde junto com } \\
\text { estratégias mais amplas } \\
\text { para apoiar seu bem-estar } \\
\text { psicológico durante e após } \\
\text { a pandemia de Covid-19. }\end{array}$ \\
\hline
\end{tabular}




\begin{tabular}{|c|c|c|c|c|c|}
\hline El-Hage et al. & $\begin{array}{l}\text { Fornecer } \\
\text { informações } \\
\text { atualizadas sobre os } \\
\text { riscos potenciais } \\
\text { para a saúde mental } \\
\text { associados à } \\
\text { exposição de } \\
\text { profissionais de } \\
\text { saúde à pandemia } \\
\text { Covid-19. }\end{array}$ & 06 estudos. & $\begin{array}{l}\text { Revisão narrativa } \\
\text { identificando } \\
\text { resultados } \\
\text { relevantes na } \\
\text { literatura científica } \\
\text { e médica } \\
\text { considerando as } \\
\text { epidemias } \\
\text { anteriores de } 2003 \\
\text { (SARS-CoV-1) e } \\
\text { 2009 (H1N1) com } \\
\text { os dados mais } \\
\text { recentes sobre a } \\
\text { pandemia do } \\
\text { Covid-19. }\end{array}$ & $\begin{array}{l}\text { As características da doença } \\
\text { da atual pandemia de } \\
\text { Covid-19 provocaram um } \\
\text { clima generalizado de } \\
\text { cautela e incerteza, } \\
\text { principalmente entre os } \\
\text { profissionais de saúde, por } \\
\text { uma série de causas, como a } \\
\text { rápida disseminação do } \\
\text { vírus, a gravidade dos } \\
\text { sintomas que pode causar } \\
\text { em um segmento de } \\
\text { infectados, o } \\
\text { desconhecimento da doença } \\
\text { e as mortes entre os } \\
\text { profissionais de saúde. }\end{array}$ & $\begin{array}{l}\text { Relatar informações como } \\
\text { essas é essencial para } \\
\text { planejar futuras estratégias } \\
\text { de prevenção, visto que a } \\
\text { proteção dos profissionais } \\
\text { de saúde é, de fato, um } \\
\text { componente importante das } \\
\text { medidas de saúde pública } \\
\text { para lidar com crises de } \\
\text { saúde em grande escala. }\end{array}$ \\
\hline $\begin{array}{l}\text { Matthew } \\
\text { Walton et al. }\end{array}$ & $\begin{array}{l}\text { Detalhar os efeitos } \\
\text { na equipe e aborda } \\
\text { algumas das } \\
\text { considerações } \\
\text { organizacionais, de } \\
\text { equipe e individuais } \\
\text { para apoiar a equipe } \\
\text { (pragmaticamente) } \\
\text { durante esta } \\
\text { pandemia. }\end{array}$ & 32 estudos. & $\begin{array}{l}\text { Revisão sistêmica } \\
\text { da bibliográfica } \\
\text { com metanálise. }\end{array}$ & $\begin{array}{l}\text { A pesquisa abordou sobre } \\
\text { os efeitos psicológicos de } \\
\text { surtos de doenças } \\
\text { infecciosas, como síndrome } \\
\text { respiratória aguda grave } \\
\text { (SARS) e gripe pandêmica } \\
\text { (H1N1), mostra padrões } \\
\text { consistentes de reações e } \\
\text { cobre as experiências da } \\
\text { equipe no trabalho, aqueles } \\
\text { em quarentena e aqueles } \\
\text { que retornam ao trabalho } \\
\text { após tempo ausente doente }\end{array}$ & $\begin{array}{l}\text { Entende-se que existem } \\
\text { oportunidades em todos os } \\
\text { níveis para fazer a } \\
\text { diferença no apoio à saúde } \\
\text { mental do pessoal e para } \\
\text { identificar e encorajar } \\
\text { oportunidades de encontrar } \\
\text { crescimento e significado } \\
\text { nesta situação. }\end{array}$ \\
\hline $\begin{array}{l}\text { Cindy Frias et } \\
\text { al. }\end{array}$ & $\begin{array}{lr}\text { Destacar } & \text { a } \\
\text { manutenção da } & \text { boa } \\
\text { saúde física e mental } \\
\text { por meio } & \text { de } \\
\text { iniciativas } & \text { que } \\
\text { melhorem } & \text { a } \\
\text { resiliência à crise, } \\
\text { como a criação de } \\
\text { Equipes } & \text { de } \\
\text { Intervenção } & \text { em } \\
\text { Saúde Mental } & \end{array}$ & 11 estudos. & $\begin{array}{l}\text { Revisão sistêmica } \\
\text { da bibliográfica } \\
\text { com metanálise. }\end{array}$ & $\begin{array}{l}\text { Os hospitais espanhóis } \\
\text { criaram comitês de } \\
\text { emergência como uma } \\
\text { estrutura de resposta para a } \\
\text { prevenção, detecção e } \\
\text { proteção de pacientes, } \\
\text { famílias e profissionais de } \\
\text { saúde e não médicos usando } \\
\text { medidas de contenção, } \\
\text { incluindo a abertura de } \\
\text { unidades de terapia } \\
\text { intensiva e a reorganização } \\
\text { de enfermarias } \\
\text { psiquiátricas. }\end{array}$ & $\begin{array}{l}\text { Nota-se a importância de } \\
\text { oferecer suporte } \\
\text { profissional especializado } \\
\text { em situações de } \\
\text { emergência usando Equipes } \\
\text { de Intervenção em Saúde } \\
\text { Mental para prevenir e } \\
\text { detectar mudanças no bem- } \\
\text { estar emocional e reduzir o } \\
\text { burnout em profissionais de } \\
\text { saúde. }\end{array}$ \\
\hline
\end{tabular}

Fonte: Pesquisa direta (2020).

\section{Discussão}

\section{O papel da equipe de enfermagem e as condições de trabalho frente a pandemia de Covid-19}

O trabalho do enfermeiro, normalmente, baseia-se nos cuidados com o indivíduo garantindo seu conforto e bem estar, atuando na prevenção e controle de infecções, na coordenação da equipe de enfermagem e dentre outras funções que fazem com que este profissional seja o qual mais tenha contato com a população em geral, atuando sempre na linha de frente contra as mais diversas doenças (Frias et al, 2020; Moreira \& Lucca, 2020).

Com relação à pandemia de Covid-19 este profissional no Brasil trabalha tanto na assistência e no cuidado com o paciente, como ainda efetuam atividades de conscientização e educação em saúde acerca da utilização adequada de EPI's, (colocar por extenso antes de colocar abreviação) na construção de áreas de isolamento e descanso, na prevenção de contaminação, na manutenção da segurança do paciente (Moreira \& Lucca, 2020).

Diante das atribuições que a equipe de enfermagem já possui, esse novo cenário faz com que surja uma preocupação mundial em ampliar o número de enfermeiros, técnicos e auxiliares de enfermagem, nas zonas epidêmicas, como por exemplo, 
a China que em 1 de março de 2020, enviou cerca de 28.000 mil enfermeiros para Hubei, província de Wuhan, para atuarem contra a infecção de Covid-19 (Catton et al, 2020).

Infere-se, portanto, que mesmo que, atualmente, o número de profissionais de enfermagem qualificados seja numeroso, cerca de 27,9 milhões, estes são mal distribuídos, o que gera escassez de mão de obra especializada em alguns locais ocasionando uma maior sobrecarga de trabalho, baixo salário, carga horaria elevada, preconceito? e limitada regulamentação trabalhista (Fofana et al, 2020).

E mesmo diante de todos esses fatores, estudos atuais revelam que a equipe de enfermagem vem sendo resiliente perante a pandemia de Covid-19, demostrando afinco e compaixão. Contudo, agentes estressores, como falta de EPIs?, a complicada conversação com os pacientes, o aumento na quantidade de pacientes, a culpa por o paciente morrer "sozinho" devido ao isolamento, a escolha de quem deve ser colocado no respirador, vêm afetando gravemente estes profissionais tanto nos âmbitos mentais como nos físicos(Catton et al, 2020; Walton et al., 2020; Frias et al, 2020).

Outro estressor, considerado um dos mais relevantes, é o risco que esses profissionais estão enfrentando de contaminação, haja a vista que durante o surto de SARS, vírus parecido com o novo coronavírus, em 2003 na China 3000 mil profissionais de saúde foram infectados nos primeiros dias da epidemia. A respeito do Covid-19, segundo a Organização Mundial da Saúde, os profissionais da saúde em geral representam $4 \%$ a $12 \%$ dos infectados, todavia, este número chegou a $50 \%$ em alguns hospitais do Reino Unido (Yu et al, 2020; Moreira \& Lucca, 2020).

No Brasil, de acordo com o Conselho Federal de Enfermagem o país possuía, em junho, 190 enfermeiros que vieram a óbito devido à infecção de Covid-19, assim se tornando o país com a maior taxa de letalidade dessa categoria de profissional no mundo. Sendo que com o aumento dos profissionais afastados devido à infecção por Covid-19, aumenta a sobrecarga do restante da equipe (Conselho Federal de Enfermagem, 2020).

\section{Os agravos psicossociais e a saúde mental dos enfermeiros pós-pandemia de Covid-19}

Primeiramente, é importante ressaltar que há poucos estudos científicos que abordam a saúde mental correlacionado com a pandemia de Covid-19 em profissionais da saúde linha de frente, os quais existem a maioria foram construídos na China e Reino Unido, portanto esses dados não podem ser generalizados completamente para outros locais, pois além das características socioculturais desses países serem diferentes, o sistema de saúde é distinto, a quantidade de infectados e isolamento social é dessemelhante (Ornel et al, 2020).

Entretanto, há um consenso na comunidade cientifica que provavelmente acorra após o coronavírus um surto de sofrimento mental, principalmente, entre os profissionais da saúde e entre a população que fez acertadamente o isolamento social. Tais implicações psicológicas poderão passar despercebidas na maioria dos casos e sessarão em curto período, mas em algumas circunstâncias conseguirão se tornar de longo prazo e acarretar em situações graves como a ideação suicida (Ornel et al, 2020; Fofana et al, 2020).

Levando em consideração este fato e o crescimento das taxas de infecção por Covid-19, demostram que o impacto dessa crise mundial pode ser comparável ao de uma guerra. É perceptível que as equipes de saúde estão passando por uma das maiores pressões físicas e psicológicas do século. Além disso, estão sofrendo ainda risco de infecção, tendo de trabalhar com novos protocolos e equipamentos de proteção individual (EPIs) que mudam frequentemente e declínio no setor de saúde (Walton et al, 2020; El-Hage et al, 2020).

Tais conjecturas podem levar ao aparecimento ou agravos psicossociais já existentes, sendo que os mais citados na literatura foram a ansiedade e a depressão, que segundo Mo et al. (2020) aumentaram principalmente enfermeiros, em especial nas mulheres, trabalhadoras na linha de frente contra o Covid-19. em Hubei, outro estudo afirma que as taxas de depressão e ansiedade entre enfermeiros de hospitais nos EUA giram em 25\% e 40\%. Tal fato é explicado por Miranda et al. (2020), 
discorrendo que esta classe de profissionais possui a maior carga horaria de trabalho, o maior contato com o paciente e menor apoio da população em geral (Ornel et al, 2020; Miranda et al, 2020; Lai et al, 2020; Mo et al, 2020; Teng et al, 2020).

Já de acordo com Sawar et al. (2020), aproximadamente, 80\% dos profissionais de saúde dos EUA demonstram alto risco de esgotamento, tal sinal pode ser a primeira manifestação clínica da síndrome Burnout, embora essa condição geralmente seja estabelecida de forma longitudinal e esteja relacionada a fatores organizacionais, a gravidade da pandemia pode desencadear esgotamento emocional, que por sua vez se correlaciona com depressão clínica, ansiedade, distúrbios do sono e uso indevido de substâncias (Sawar et al, 2020; Mo et al, 2020).

Profissionais da linha de frente também mostraram ter um risco $10 \%$ maior de desenvolver transtorno de estresse póstraumático (TEPT), que persistiu mesmo após um período de afastamento do trabalho, a mesma coisa aconteceu com profissionais de saúde expostos ao surto de SARS em 2003, sendo que este agravo pendurou de um a dois anos após o fim desse surto. $\mathrm{O}$ trauma vicário ou estresse traumático secundário, também já foi relatado na literatura com profissionais que enfrentam o Covid-19, este agravo é comum em indivíduos que enfrentam situações de catástrofes e acabam apresentando sinais e sintomas parecidos ao do paciente (Ornel et al, 2020; Walton et al, 2020; Preti et al, 2020).

Além disso, os enfermeiros de linha de frente podem sofrer estigmas pela população assistem pacientes com alto potencial de contágio. Por outro lado, também podem receber o status de super-heróis o que acarreta prestigio, mas também pressão social, pois heróis não erram, não adoecem e não desistem. Isto demarca a necessidade de apoio emocional, incentivo e valorização para estes profissionais (Ornel et al, 2020; Choudhury et al, 2020).

Eles também podem sofrer após a pandemia de tédio, exaustão e solidão, podem estar receosos ou ansiosos por voltar ao trabalho. Contudo, é importante ressaltar que nem todos os agravos relatados poderão ocorrer, haja a vista, que a pandemia ainda é algo recente e não existe na literatura estudos pertinentes sobre o assunto, sugere-se, portanto, estudos adicionais para analisar a saúde mental e as consequências neuropsiquiátricas pós-pandemia de Covid-19.

\section{O suporte a saúde mental dos profissionais de saúde}

O combate a Covid-19, dentro dos centros de saúde, demanda de uma diversificada equipe multiprofissional que inclui os trabalhadores da saúde e serviços de reforço, sendo categorias com associação empregatícia, carga horária e jornadas de trabalho diversificadas. Dentre as classes supracitadas, os Profissionais de Enfermagem (PE), público de interesse da presente revisão (Moura et al, 2020).

Em vista disso, os PE precisam de amparo no enfrentamento da Covid-19 na rede pública e privado de saúde, dispondo de apoio governamental e das chefias, acesso a materiais esterilizados e Equipamento de Proteção Individual (EPI), recebam workshops sobre capacitação no atendimento em urgência e emergência de infectados, obtenham orientações da instituição de saúde com um Protocolo Operacional Padrão (POP) de controle de infecções e manejo de contaminados com auto potencial para disseminação do novo coronavírus, assim como, normas atualizadas da referência dos pacientes, sendo recursos para assegurar o profissional exposto ao surto (Moreira \& Lucca, 2020).

A curva de contágio permanece aumentando, por isso, as iniciativas de saúde para as equipes no embate da pandemia também devem ser reforçadas. Em caso de negligência ou falta de priorização, além de um possível colapso do sistema de saúde, os profissionais estão sujeitos a sofrer uma crise emocional. Contudo, apesar das desigualdades socioeconômicas no Brasil, o país possui um Serviço Único de Saúde (SUS) com direito a universalidade e equidade dos seus usuários. Assim, os profissionais esgotados mentalmente devem ser assistidos, independente das fragilidades reveladas em momentos de crise (Ornell et al, 2020).

Dessa maneira, o suporte psicossocial em saúde mental do trabalhador ganha evidência, nas ações de prevenção, promoção e recuperação das pessoas com transtornos mentais. Considerando necessário proporcionar uma sensação de 
pertencimento ao profissional, uma sensação de ressignificação do seu valor no local de trabalho e, principalmente, valorizando o seu protagonismo em meio ao atual cenário. Tais condutas fortalecem o emocional, bem-estar psíquico, aumenta da autoestima e valorização da vida, pois a rotina no ambiente de trabalho detém momentos de contentamento e frustração, sendo importante a união entre as equipes.

Logo, a atenção à saúde mental dos profissionais não pode ser minimizada no pós-pandemia, visto que alguns indivíduos possuem dificuldade em exteriorizar ou partilhar com alguém o esgotamento físico e mental, as incertezas, os medos, as angústias, os desapontamentos e, até mesmo, o luto. Dessa forma, é fundamental que haja o estabelecimento de estratégias efetivas para cada estágio do surto, as quais seriam adaptadas as necessidades de cada um.

A fim de atender à essa problemática, vários meios surgiram para prestar uma tenção psicológica aos profissionais de saúde, a exemplo disso temos o material midiático "Uma mensagem para os profissionais da saúde", vídeo disponibilizado pelo Ministério da Saúde (MS). Ademais, nos últimos meses, foi criado um canal telefônico para teleconsulta exclusiva a estes trabalhadores, com mais de 10 mil horas de atendimento psicológico, estando disponível no site do Conselho Federal de Enfermagem (Cofen) e Conselhos Regionais de Enfermagem (Coren), bem como, a Rede Cuidar Enfermagem e Universidade Estadual do Pará (UEPA) que oferece escuta segura gratuita em Práticas Integrativas e Complementares (PIC) (Blake et al, 2020).

Essas intervenções têm como finalidade de manutenção de uma boa qualidade de vida dos profissionais, melhorando sua resiliência mediante esta crise. Por isso, é valido ofertar suporte profissional especializado com Equipes de Intervenção em Saúde Mental (EISM), prevenindo ou detectando precocemente alterações de humor, sinais depressivos, ansiedade patológica e a síndrome do Burnout (Cindy et al, 2020).

Esse estudo apresentou limitações relacionadas a quantidade de estudos encontrados, sendo evidente um déficit de publicações relacionadas ao tema proposto, considerando uma concentração do corte temporal no ano de 2020, a escassez de literatura brasileira e a restrição da problemática a equipe de enfermagem, desconsiderando as demais classes de profissionais da saúde que estão na linha de frente ao combate do novo coronavírus.

\section{Conclusão}

A partir das considerações apresentadas no decorrer do estudo, notou-se que a equipe de enfermagem está vulnerável para desenvolver frustrações e inseguranças no trabalho, bem como, encarar a falta de apoio dos colegas de equipe, chefia ou coordenadores e da instituição filiada, gerando descontentamento e o desejo súbito de abandonar a profissão.

Diante disso, faz-se ímpar o papel da enfermagem em todas as redes de serviços em saúde, seguindo para fora dos muros de unidades básicas e hospitais, visando o seu potencial nas estratégias de educação em saúde, por isso, o apoio dos sujeitos supracitados, além das entidades governamentais em parceria com os conselhos de enfermagem, tendo como principal objetivo a preservação e garantia da saúde mental desses profissionais para que tenham o bem-estar durante o cuidado do próximo.

Assim, o elo na corrente multiprofissional no combate a Covid-19, prioriza a vida humana, atentando para o apoio psicossocial do trabalhador e a segurança do paciente. Em decorrência do cenário desafiador vivenciado, deve ocorrer uma reinvenção da sociedade e um alinhamento dos setores populacionais, em busca de associar classes indispensáveis.

Compreende-se que atividades relacionadas ao Covid-19 terão amplitude acentuada nos próximos anos, é necessário acompanhar as intervenções desenvolvidas em cada setor de saúde, seguindo desde a rede de atenção básica até os serviços de ala hospitalar, observando as contribuições de caráter transdisciplinar para a problemática. Desse modo, como agenda de pesquisa alternativa, sugerem-se as seguintes indagações: a) analisar os benefícios e deletérios da Covid-19 no psicológico da equipe de enfermagem; b) observar os agravos psicossociais e inadequações nos atendimentos de profissionais com agravos 
psicossociais na pandemia; c) avaliar a eficácia do suporte oferecido a saúde desses profissionais, assim como, a articulação desta assistência.

\section{Referências}

Blake, H., Bermingham, F., Johnson, G., \& Tabner, A. (2020). Mitigating the psychological impact of Covid-19 on healthcare workers: a digital learning package. Res Public Health, 17(9).

Choudhury, T., Debski, M., Wiper, A., Abdelrahman, A., Wild, S., Chalil, S., More, R., Goode, G., Patel, B., \& Abdelaziz, H. K. (2020). Covid-19 pandemic: looking after the mental health of our healthcare workers. Journal Occupacional And Environ Medicine, 12(1).

El-Hage, W. (2020). Les professionnels de santé face à la pandémie de la maladie à coronavirus (Covid-19): quels risques pour leur santé mentale? Encephale, 46(3), 73-80.

Fofana, N. K., Latif, F., Sarfraz, S., Bilal, B. M. F., \& Komal, B. (2020). Fear and agony of the pandemic leading to stress and mental illness: an emerging crisis in the novel coronavirus (Covid-19). Psychiatry Research, 2(10).

Frias, C. E. (2020). Resilience and emotional support in health care professionals during the covid-19 pandemic. Journal Of Psychosocial Nursing and Mental Health Services, 58(6), 5-6.

Lai, J., Ma, S., Wang, Y., Cai, Z., Hu, J., Wei, N., Wu, J., Du, H., Chen, T., Li, R., Tan, H., Kang, L., Yao, L., Huang, M., Wang, H., Wang, G., Liu, Z., \& Hu, S. (2019). Factors associated with mental health outcomes among health care workers exposed to coronavirus disease 2019. Jama Netw Open, 3(3).

Liu, Y. E. (2020). Experiences of front-line nurses combating coronavirus disease-2019 in china: a qualitative analysis. Public Health Nurs, 37(2), 757-763.

Miranda, F. M. A. (2020). Condições de trabalho e o impacto na saúde dos profissionais de enfermagem frente a Covid-19. Cogitare Enfermagem, 25(1).

Moreira, A. S., \& Lucca, S. R. (2020). apoio psicossocial e saúde mental dos profissionais de enfermagem no combate à Covid-19. Enfermagem Em Foco, $11(1), 155-161$.

Mo, Y., Deng, L., Zhang, L., Lang, Q., Liao, C., Wang, N., Qin, M., \& Huang, H. (2020). Work stress among chinese nurses to support wuhan in fighting against Covid-19 epidemic. Journal Nurses Manag, 28(5).

Ornel, F. (2020). The impact of the covid-19 pandemic on the mental health of healthcare professionals. Cadernos De Saúde Pública, 36(4).

Preti, E., Di, M. V., Perego, G., Ferrari, F., Mazzetti, M., Taranto, P., Di, P. R., Madeddu, F., \& Calati, R. (2020). The psychological impact of epidemic and pandemic outbreaks on healthcare workers: rapid review of the evidence. Curr Psychiatry Rep, 10(22), 38-43.

Santarone, K., Mckenney, M., \& Elkbuli, A. (2020). Preserving mental health and resilience in frontline healthcare workers during Covid-19. Am J Emerg Medicine, 38(7), 1530-1531.

Sawar, M. A. A., \& Sawar, H. (2020). The impact of Covid-19 on the mental health of healthcare professionals. Journal Of College Of Physicians And Surgeons Pakistan, 30(2), 583-584.

Teng, Z., Huang, J., Qiu, Y., Tan, Y., Zhong, Q., Tang, H., Wu, H., Wu, Y., \& Chen, J. (2020). Mental health of front-line staff in prevention of coronavirus disease 2019. Zhong Nan Da Xue Bao Yi Xue Ban, 28(45), 613-619.

Walton, M., Murray, E., \& Christian, M. D. (2020). Mental health care for medical staff and affiliated healthcare workers during the Covid-19 pandemic. Eur Heart J Acute Cardiovasc Care. 9(3), 241-247. 